\title{
Polycystin-1, the PKD1 gene product, is in a complex containing E-cadherin and the catenins
}

\author{
Yonghong Huan and Janet van Adelsberg \\ Department of Medicine, Columbia University, New York, New York 10032, USA \\ Address correspondence to: Janet van Adelsberg, Department of Medicine, Columbia University, 630 West 168th Street, Box 84, \\ New York, New York 10032, USA. Phone: (212) 305-4476; Fax: (212) 305-3475; E-mail: jsv1@ columbia.edu.
}

Received for publication September 2, 1998, and accepted in revised form October 5, 1999.

\begin{abstract}
Autosomal dominant polycystic kidney disease (ADPKD) is a common human genetic disease characterized by cyst formation in kidney tubules and other ductular epithelia. Cells lining the cysts have abnormalities in cell proliferation and cell polarity. The majority of ADPKD cases are caused by mutations in the PKD1 gene, which codes for polycystin-1, a large integral membrane protein of unknown function that is expressed on the plasma membrane of renal tubular epithelial cells in fetal kidneys. Because signaling from cell-cell and cell-matrix adhesion complexes regulates cell proliferation and polarity, we speculated that polycystin-1 might interact with these complexes. We show here that polycystin-1 colocalized with the cell adhesion molecules E-cadherin and $\alpha$-, $\beta$-, and $\gamma$-catenin. Polycystin- 1 coprecipitated with these proteins and comigrated with them on sucrose density gradients, but it did not colocalize, coprecipitate, or comigrate with focal adhesion kinase, a component of the focal adhesion. We conclude that polycystin- 1 is in a complex containing E-cadherin and $\alpha$-, $\beta$-, and $\gamma$-catenin. These observations raise the question of whether the defects in cell proliferation and cell polarity observed in ADPKD are mediated by E-cadherin or the catenins.
\end{abstract}

J. Clin. Invest. 104:1459-1468 (1999).

\section{Introduction}

Autosomal dominant polycystic kidney disease (ADPKD) is one of the most common genetic diseases in humans. It is a multisystem disease that causes renal cysts and renal failure as well as cysts in the liver, pancreas, and other organs, aneurysms of the cerebral and other arteries, cardiac valvular insufficiency, and colonic diverticula (1).

ADPKD is caused by mutations in at least 3 genes. Two of these, PKD1 and PKD2, have been cloned and sequenced (2-5). Families with ADPKD unlinked to $P K D 1$ or $P K D 2$ have been identified, so at least 1 more locus must exist (6-9). Both PKD1 and PKD2 code for novel molecules whose biochemical functions are unknown. The PKD1 gene product, polycystin-1, is a large intrinsic membrane protein with a molecular weight of approximately $485 \mathrm{kDa}$ that is expressed in a developmentally regulated manner. The amino terminal domain of polycystin- 1 is predicted to be extracytoplasmic. The carboxyl terminal domain of polycystin- 1 is predicted to have between 7 and 11 transmembrane helices. Polycystin-2, the PKD2 gene product, is an intrinsic membrane protein with a molecular weight of $110 \mathrm{kDa}$. It is predicted to have intracellular amino and carboxyl termini and has significant similarities to transient receptor potential (Trp) channel subunits. The observation that carboxyl terminal fragments of polycystin- 1 and -2 interact in vitro and in transfected cells, coupled with the similarity of the disease caused by mutations in either PKD1 or $P K D 2$, suggests that these molecules act together in an uncharacterized biochemical pathway $(10,11)$.

The primary structure of polycystin-1 suggests that it might be a receptor. The large extracellular domain begins with 2 leucine-rich repeats and has 16 novel repeats with an Ig-like fold, an LDL-A domain, a calci- um-dependent lectin domain, and a domain of nearly 1,000 amino acids that is similar to the sea urchin receptor for egg jelly. These domains mediate protein-protein and protein-carbohydrate interactions in other proteins, and by analogy, are likely to mediate similar interactions in polycystin-1. We and others have shown that polycystin-1 is expressed in the plasma membrane of epithelial cells (12-17). This observation is consistent with the hypothesis that polycystin-1 might interact with an extracellular ligand or ligands.

We had observed that polycystin-1 was concentrated on the apical and lateral aspects of epithelial cells in developing kidneys (12). This localization pattern was similar to that of the cell adhesion molecule E-cadherin in developing kidneys, and suggested that polycystin-1 might interact with E-cadherin. This hypothesis was particularly intriguing because of the roles that E-cadherin plays in orchestrating cell proliferation and polarization of epithelial monolayers. E-cadherin modulates proliferation and polarity through associated cytoplasmic polypeptides, the catenins (18-20). E-cadherin regulates cell proliferation and gene expression by participating in the regulation of $\beta$-catenin metabolism $(21,22)$. Cyst-lining epithelial cells in ADPKD express markers for cell proliferation in vivo and are abnormally sensitive to growth factors in vitro, suggesting that there is a derangement of the control of cell growth in ADPKD (23). E-cadherin orchestrates cell polarity by directing a cytoskeletal network containing ankyrin and spectrin to the basolateral aspect of the epithelial cell $(24,25)$. Na,KATPase binds to ankyrin and is retained in the basolateral membrane by its interaction with the ankyrin/spectrin cytoskeleton $(26,27)$. When these complexes are disrupted, for example by ischemia, Na,K-ATPase 
becomes depolarized and appears in both lateral and apical plasma membranes $(28,29)$. Some, but not all, investigators have found that $\mathrm{Na}, \mathrm{K}$-ATPase is depolarized in ADPKD epithelia (30-32). These observations suggested that polycystin-1 might modulate cell proliferation and cell polarity by interacting with E-cadherin or the catenins. We found that polycystin-1 is part of a complex containing E-cadherin and the catenins, suggesting that some of the defects observed in ADPKD might be mediated by E-cadherin or the catenins.

\section{Methods}

Antibodies. Rabbit polyclonal antibody against polycystin peptide B145 has been described previously (12). Mouse monoclonal antibodies against human $\gamma$-catenin were obtained from Zymed Laboratories (South San Francisco, California, USA) and from Transduction Laboratories (Lexington, Kentucky, USA). Mouse monoclonal antibodies against human $\beta_{1}$-integrin was obtained from GIBCO BRL (Gaithersburg, Maryland, USA) and Transduction Laboratories. Monoclonal antibodies against human E-cadherin, $\alpha$ - and $\beta$-catenin, and focal adhesion kinase (FAK) were obtained from Transduction Laboratories. Cy3 and horseradish peroxidase-labeled secondary antibodies absorbed against human, rat, mouse, and rabbit serum proteins were obtained from Jackson ImmunoResearch Laboratories Inc. (West Grove, Pennsylvania, USA). Oregon Green 488-labeled secondary antibodies were obtained from Molecular Probes Inc. (Eugene, Oregon, USA).

Immunofluorescence. Indirect immunofluorescence on frozen sections was performed as described previously (12). Briefly, the sections were fixed in methanol and acetone $(1: 1, \mathrm{vol} / \mathrm{vol})$ at $-20^{\circ} \mathrm{C}$ for 10 minutes. The sections were air dried, rehydrated in PBS, and blocked for 15-30 minutes in $0.2 \%$ (wt/vol) BSA in PBS. Primary antibodies were diluted in blocking buffer containing the following: polycystin-1, either 1:10 (affinity purified) or 1:50 (anti-serum); E-cadherin, $1: 25 ; \alpha$-catenin, $1: 5 ; \beta$ catenin, $1: 50$; $\gamma$-catenin (plakoglobin), 1:100; FAK, 1:5; and $\beta_{1}$-integrin, $1: 5$. Human fetal kidneys of $20-24$ weeks gestational age were obtained under a protocol approved by the Institutional Review Board of Columbia University. The sections were postfixed in $4 \%$ paraformaldehyde before being mounted for confocal microscopy in Prolong Antifade (Molecular Probes Inc.).

Confocal microscopy. Confocal microscopy was performed with a Zeiss LSM 410 laser scanning on a Zeiss Axiovert 100 microscope platform using an argon-krypton laser (Carl Zeiss North America, Thornwood, New York, USA). The 488-nm and 568-nm laser lines were used for Oregon Green and Cy3 detection, respectively. Images were collected with a 515-540-nm band pass filter (green channel) and a 590-nm long pass filter (red channel). Sections were scanned at low power on the red channel (polycystin-1 staining) to select tubules strongly stained for polycystin. These tubules were then examined at high power with both red and green channels. Red and green images were collected simultaneously and stored for analysis as both individual red or green images and as the dual-scanned image. For each image, tissue sections were viewed at $\times 100$ or $\times 40$. The images $(512 \times$
512 pixels) were projected at 600 pixels/inch using Adobe Photoshop 4.0 (Adobe Systems Inc., Mountain View, California, USA), which was also used to make the montages shown in Figures 1 and 5.

Cell lines. The human pancreatic adenocarcinoma (HPAC) cell line was obtained from the American Type Culture Collection (Rockville, Maryland, USA). Cells were grown in a 1:1 mixture of DMEM and Ham's F12 medium containing $1.2 \mathrm{~g} / \mathrm{L}$ sodium bicarbonate, $15 \mathrm{mM}$ HEPES, $0.002 \mathrm{mg} / \mathrm{mL}$ insulin, $0.005 \mathrm{mg} / \mathrm{mL}$ transferrin, $40 \mathrm{ng} / \mathrm{mL}$ hydrocortisone, and $10 \mathrm{ng} / \mathrm{mL}$ EGF, with 5\% FBS (HyClone Laboratories, Logan, Utah, USA). Insulin, transferrin, and EGF were purchased from Fisher Scientific (Atlanta, Georgia, USA). DMEM, F12, and HEPES were purchased from GIBCO BRL. Hydrocortisone was obtained from Sigma Chemical Co. (St. Louis, Missouri, USA).

RT-PCR. Total RNA was prepared from cultured cells using RNAzol B (Tel-Test Inc., Friendswood, Texas, USA). Primers for the $3^{\prime}$ end of the PKD1 gene were selected using the Prime program (Wisconsin Package, version 8.0) from Genetics Computer Group Inc. (Madison, Wisconsin, USA). The forward primer ( $5^{\prime}$ to $\left.3^{\prime}\right)$ sequence was GGC TGT TAT TCT CCG CTG (nucleotides 12,441-12,458, HSU24497). The reverse primer $\left(5^{\prime}\right.$ to $\left.3^{\prime}\right)$ sequence was GGG TGG ACC TTG TTC TTG (nucleotides 13,034-13,017). RT-PCR was carried out using the Access RT-PCR System from Promega Corp. (Madison, Wisconsin, USA), according to the manufacturer's instructions, except that $10 \%$ DMSO was included in the reaction. Human $\beta$-actin primers from 2 exons (Stratagene, La Jolla, California, USA) were used as a positive control; RNA treated with RNase A (Sigma Chemical Co.) was used as a negative control. RT-PCR products were separated on a $3 \%$ NuSieve 3:1 agarose gel (FMC BioProducts, Rockland, Maine, USA) in $1 \times$ Trisacetate-EDTA buffer, and were purified using QIAEX resin (QIAGEN Inc., Valencia, California, USA) for direct sequencing at the DNA facility at Columbia University.

Immunoprecipitation and immunoblotting. HPAC cells were labeled overnight with $\left[{ }^{35} \mathrm{~S}\right]$ methionine/cysteine (EXPRESS label; NEN Life Science Products, Inc., Boston, Massachusetts, USA) at $0.1 \mathrm{mCi} / \mathrm{mL}$ in methionine/cysteine-free medium with dialyzed FBS. Cells were suspended in lysis buffer containing $1 \%$ (vol/vol) Triton $\mathrm{X}-100,0.5 \%$ (vol/vol) NP-40, $150 \mathrm{mM} \mathrm{NaCl}, 10 \mathrm{mM}$ Tris$\mathrm{HCl}$ (pH 7.4), $1 \mathrm{mM}$ EDTA, $1 \mathrm{mM}$ EGTA, $0.2 \mathrm{mM}$ $\mathrm{Na}_{3} \mathrm{VO}_{4}, 1 \mathrm{mM}$ PMSF, $2 \mu \mathrm{g} / \mathrm{mL}$ aprotinin, $1 \mu \mathrm{g} / \mathrm{mL}$ E-64, $10 \mu \mathrm{g} / \mathrm{mL}$ leupeptin, and $1 \mu \mathrm{g} / \mathrm{mL}$ pepstatin A. Cells were lysed by passage through a 22-gauge needle several times. Insoluble material was removed by centrifugation at $10,000 \mathrm{~g}$ for 30 minutes at $4^{\circ} \mathrm{C}$. Lysates were precleared by incubation with preimmune rabbit serum and protein A-agarose (Oncogene Science Inc., Uniondale, New York, USA) for 30 minutes at $4^{\circ} \mathrm{C}$. After removal of protein $\mathrm{A}$-agarose by centrifugation, lysates were incubated for 1 hour at $4{ }^{\circ} \mathrm{C}$ with equivalent concentrations of protein A-purified IgG from preimmune serum, immune serum from rabbits immunized with peptide B145, or immune serum plus peptide B145 at 0.4 $\mathrm{mg} / \mathrm{mL}$. For most experiments, antibody to peptide B145 was purified on a B145 peptide column as described previously (12). Affinity-purified antibody was 
used at a concentration of $1-5 \mu \mathrm{g} / \mathrm{mL}$. After 1 hour, protein $\mathrm{A}$-agarose was added and the lysates were incubated for an additional 30 minutes. Immunoprecipitates were washed 4 times with lysis buffer and then boiled in SDS-PAGE sample buffer. Cleared cell lysates and immunoprecipitates were separated on a $4-10 \%$ linear gradient polyacrylamide gel and examined by autoradiography using a PhosphorImager from Molecular Dynamics (Sunnyvale, California, USA).

For coprecipitation experiments, unlabeled HPAC cell lysates were prepared as above, except that affinity-purified antibody to peptide B145 was used for all experiments. Cell lysates and immunoprecipitates were separated on a $4-15 \%$ gradient gel, transferred to nitrocellulose, and probed with mouse monoclonal antibodies against different components of cell adhesion complexes using an HRPlabeled anti-mouse secondary antibody (Jackson ImmunoResearch Laboratories Inc.) and enhanced chemiluminescence (Amersham Pharmacia AB, Uppsala, Sweden) to detect antibody

\section{Figure 1}

Confocal images of sections of human fetal kidney subjected to double-stain indirect immunofluorescence with antibodies to polycystin-1 (red) and to junctional proteins (green). Each horizontal series shows, from left to right, the green channel (junctional protein), the red channel (polycystin-1), and the total fluorescence (dual-scanned image). Colocalization is seen in the right-hand panels as yellow staining. (a-c) Double staining for polycystin-1 and Ecadherin, where (a) is E-cadherin, (b) is polycystin-1, and (c) is the dual-scanned image. $(\mathbf{d}-\mathbf{f})$ Double staining for $\alpha$-catenin, where (d) is $\alpha$-catenin, (e) is polycystin- 1 , and ( $\mathbf{f})$ is the dual-scanned image. ( $\mathbf{g}-\mathbf{i})$ Double staining for $\beta$-catenin, where $(\mathbf{g})$ is $\beta$-catenin, $(\mathbf{h})$ is polycystin-1, and (i) is the dual-scanned image. (j-I) Double staining for $\gamma$-catenin, where $(j)$ is $\gamma$ catenin, (k) is polycystin-1, and (I) is the dualscanned image. In all these images, polycystin1 and E-cadherin or the catenins are colocalized (yellow) at the apical and lateral aspects of the epithelial cells. There are areas where distinct green or red staining can be seen, showing that not all of the polycystin- 1 colocalizes with the junctional proteins. These images were acquired with the $\times 100$ lens. $(\mathbf{m}-\mathbf{o})$ Confocal images of antibodies to polycystin-1 (red) and $\beta$-catenin (green) incubated in the presence of the peptide against which the polycystin- 1 antibody was raised. The peptide blocked polycystin-1 staining ( $\mathbf{n}$ ) but did not affect $\beta$-catenin staining ( $\mathbf{m}$, $\mathbf{o})$. Some precipitated anti-rabbit antibody is seen as red dots $(\mathbf{n})$. These images were acquired with the $\times 40$ lens. binding to the nitrocellulose membrane as described previously. Antibodies were used at the following dilutions: E-cadherin, $1: 500 ; \alpha$-catenin, $1: 500 ; \beta$-catenin, $1: 1,000 ; \gamma$-catenin (plakoglobin), 1:1,000; and FAK, 1:1,000. The HRP-labeled secondary antibodies were used at 1:10,000 to 1:50,000, depending on the lot.

For double immunoprecipitation experiments, metabolically labeled HPAC cell lysates were prepared and cleared as described above, except that both protein A and recombinant protein G-agarose (Roche Molecular Biochemicals, Mannheim, Germany) were used during the
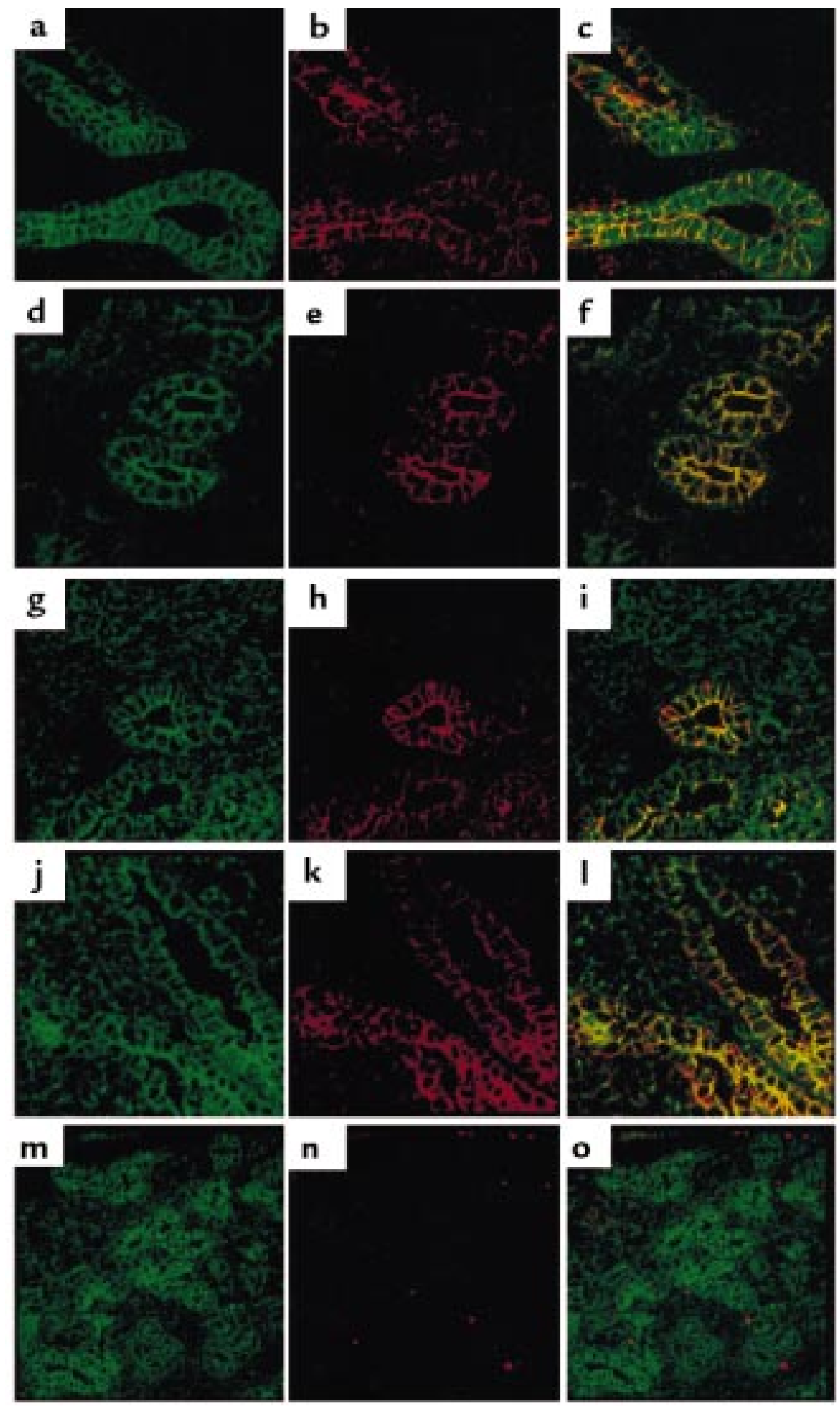


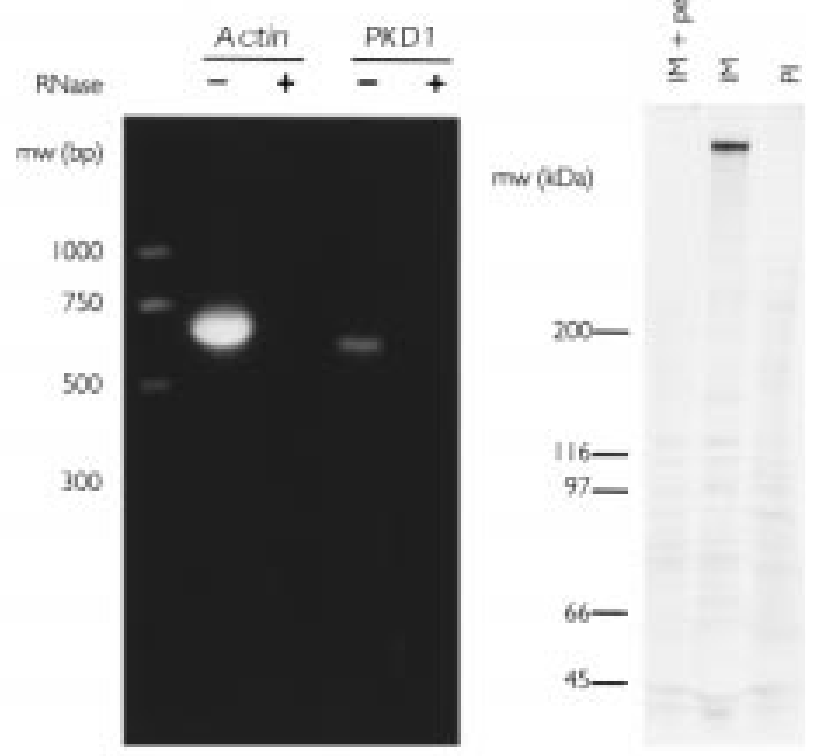

\section{Figure 2}

Expression of polycystin-1 in HPAC cells. (a) RT-PCR with PKD1-specific primers yielded the predicted 564-bp product (PKD1, - RNase). RT-PCR with human $\beta$-actin primers from 2 exons yielded the mRNA-specific product of $661 \mathrm{bp}$ (actin, - RNase). The sizes of the markers on the left are 1,000, 700, 500, and $300 \mathrm{bp}$. Treatment of the RNA template with RNase A before RT-PCR eliminated both the actin and the PKD1 products (+ RNase). (b) Polycystin-1 was precipitated from [ ${ }^{35}$ S ]methionine/cysteine-labeled HPAC cells as described, displayed on a $4-10 \%$ linear gradient gel, and viewed with a Phosphorlmager analysis system (Molecular Dynamics). IM + peptide: precipitation with immune serum in the presence of the immunizing peptide. IM: precipitation with immune serum. PI: precipitation with preimmune serum. Note that the polycystin polypeptide of approximately $500 \mathrm{kDa}$ is precipitated only by immune serum and only in the absence of peptide.

clearing step. Monoclonal antibodies to E-cadherin or $\beta$-catenin were precipitated with protein $G$-agarose in the first immunoprecipitation. The precipitates were washed 3 times with $1 \mathrm{~mL}$ lysis buffer and the precipitated proteins were released with $1 \% \operatorname{SDS}$ at $37^{\circ} \mathrm{C}$ for 30 minutes. The eluate was diluted to a final concentration of $0.1 \%$ SDS with lysis buffer, and was reprecipitated with affinity-purified antibody to peptide B145 as described above.

To estimate the molar ratio of polycystin- 1 to E-cadherin and $\beta$-catenin in precipitated complexes, the results of PhosphorImager analysis (Molecular Dynamics) were normalized to the number of methionines and cysteines in each molecule. The molar ratio of E-cadherin to $\beta$-catenin obtained by this method was $1: 1$ (range $0.7-1.1)$, agreeing with previous results $(33,34)$.

Sucrose density gradient centrifugation. HPAC cell lysates prepared as above were layered onto linear 5-20\% (wt/vol) sucrose gradients in lysis buffer and centrifuged in an SW-40 rotor (Beckman Instruments Inc., Fullerton, California, USA) at $100,000 \mathrm{~g}$ for 24 hours at $4^{\circ} \mathrm{C}$. Twenty-four fractions $(0.5 \mathrm{~mL}$ each) were collected from each gradient. Ten-microliter aliquots of each fraction were used for immunoblotting. The remainder of each fraction was immunoprecipitated with affinity-purified antibody against polycystin-1. The samples were separated on a $7.5 \%$ gel, transferred to PVDF (Millipore Corp., Bedford, Massachusetts, USA), and probed with mouse monoclonal antibodies against $\beta$-catenin. The blots were then stripped and reprobed with antibodies to FAK. Densitometry was performed on a densitometer from Molecular Dynamics.

To determine the distribution of polycystin-1 in gradients, metabolically labeled cell lysates were subjected to density gradient centrifugation as above. Ten-microliter fractions were separated on a $10 \%$ gel and transferred to PVDF. This was cut into strips at the $66 \mathrm{kDa}$ molecular weight marker, and probed with antibodies to $\beta$-catenin and actin (Roche Molecular Biochemicals). The remainder of each fraction was immunoprecipitated with affinity-purified antibody to polycystin- 1 and separated on a $7.5 \%$ gel. Autoradiograms of the immunoprecipitates were viewed with a Storm PhosphorImager System from Molecular Dynamics.

\section{Results}

Polycystin colocalizes with E-cadherin and the catenins. We and others had previously found that polycystin-1 was most highly expressed in renal epithelial cells of fetal kidney $(12,35)$. By indirect immunofluorescence, polycystin-1 was inconsistently detectable in the collecting duct of adult kidney. To test the hypothesis that polycystin interacts with complexes containing E-cadherin, we stained sections of fetal kidney with antibodies to polycystin-1 and E-cadherin. We selected tubules that were strongly stained for polycystin and then scanned those tubules for simultaneous localization of both polycystin-1 and cell adhesion molecules. The majority of the tubules were located just below the nephrogenic zone in the developing kidneys examined. The staining pattern and morphology of the tubules suggested that the majority were of ureteric bud origin. Figure 1 shows that antibodies to E-cadherin (Figure 1a, green) and polycystin-1 (Figure 1b, red) both stained the plasma membrane of tubular epithelial cells. Figure 1c shows that the majority of polycystin-1 colocalized with E-cadherin (yellow), suggesting that the 2 proteins might be part of a complex. There was a significant amount of E-cadherin in the apical plasma membrane of the tubule because this fetal tubule is not yet completely polarized. Polycystin-1 and E-cadherin were colocalized in the apical and lateral plasma membrane. There were areas where polycystin1 and E-cadherin did not colocalize, shown as distinct green or red staining (Figure 1c). These data suggest that there are pools of E-cadherin and polycystin-1 in the plasma membrane that do not interact.

The cytoplasmic domain of E-cadherin interacts with cytoplasmic molecules known as the catenins. These molecules both regulate the adhesive function of E-cadherin and mediate signaling from E-cadherin. The observation that polycystin-1 colocalized with E-cadherin suggested that polycystin-1 should also colocalize with the catenins. Distinct complexes containing E-cadherin and subsets of the catenins have been identified in epithelial cell cultures, suggesting that these complexes might have different signaling properties. The common components of these complexes were E-cadherin and $\alpha$-catenin; $\beta$-catenin and 
$\gamma$-catenin were found in a mutually exclusive manner in these complexes. It was possible that polycystin- 1 might be identified in only 1 type of E-cadherin complex. Accordingly, we stained sections of fetal kidney with antibodies to $\alpha$-, $\beta$-, and $\gamma$-catenin and to polycystin-1. We found that polycystin- 1 colocalized with $\alpha$-catenin (Figure $1, \mathrm{~d}-\mathrm{f}$ ), $\beta$-catenin (Figure $1, \mathrm{~g}-\mathrm{i}$ ), and $\gamma$-catenin (Figure $1, j-1)$. There was no difference in the location or amount of polycystin- 1 associated with $\beta$ - and $\alpha$-catenin, suggesting that polycystin- 1 did not preferentially interact with a subset of E-cadherin complexes. All 3 catenins localized to the apical and lateral plasma membranes (Figure 1, d, g, and j), paralleling the apicolateral distribution of E-cadherin in these immature epithelia. All 3 catenins were expressed in mesenchymal cells as well as epithelial cells of the developing kidney. The expression of $\beta$ - and $\gamma$-catenin was quite high and is easily seen (Figure 1, g and $\mathrm{j}$ ), whereas the expression of $\alpha$-catenin was lower and is not well seen in this figure (Figure 1d). As we had observed for Ecadherin, the association of polycystin-1 with the catenins was not complete. There were areas where polycystin-1 did not colocalize with the catenins, seen as distinct areas of green or red staining (Figure 1, f, i, and l).

To demonstrate the specificity of polycystin-1 staining, we incubated peptide B145 (the antigen against which the polycystin-1 antibody was raised) with the primary antibodies during immunostaining. The result of a representative experiment is shown in Figure 1, m-o. Peptide abolished polycystin-1 staining (Figure $1 \mathrm{n}$ ) but had no effect on $\beta$-catenin staining (Figure $1, \mathrm{~m}$ and $\mathrm{o}$ ). These data show, first, that polycystin-1 staining was peptide specific. Second, the peptide had no effect on $\beta$-catenin staining, demonstrating that there were no nonspecific effects of peptide on antibody reactivity. Third, these images show that there was excellent optical separation between the red channel (polycystin-1) and the green channel (in this case, $\beta$-catenin), because no $\beta$-catenin staining was found in the red channel.

HPAC pancreatic epithelial cells express polycystin-1. To determine whether polycystin-1 interacts with E-cadherin and the catenins, we sought a human epithelial cell line that expressed all the proteins of interest. Polycystin1 is expressed in the pancreatic ductal epithelium (van Adelsberg, unpublished observations, and ref. 17), suggesting that pancreatic ductal cell lines would be an appropriate in vitro model system. Patients with ADPKD develop pancreatic cysts, as do mice with a targeted mutation in the PKD1 gene (36). Because the disease is manifested in the pancreatic duct in vivo, cell lines derived from the pancreatic duct should be appropriate for studying the relevant biochemical interactions of polycystin-1 in vitro. We identified a human pancreatic epithelial cell line, HPAC, that grows as a monolayer and expresses the proteins E-cadherin, $\alpha$-, $\beta$-, and $\gamma$-catenin, $\beta_{1}$ integrins, and FAK (see Figure 3 ). We screened this cell line for $P K D 1$ gene and protein expression.

To determine whether the PKD1 gene was expressed in HPAC cells, we performed RT-PCR on total RNA isolated from these cells. Figure $2 \mathrm{a}$ shows that primers from the unique 3 ' end of PKD1 amplified the expected product of $564 \mathrm{bp}$. This product was purified and sequenced; the sequence was identical to the pub- lished PKD1 sequence. Primers from 2 exons of the human $\beta$-actin gene amplified the expected product of $661 \mathrm{bp}$. There was no larger product, suggesting that there was no contamination by genomic DNA. Treatment of the RNA template with RNase A before RT-PCR eliminated both the PKD1 and the actin PCR products (Figure 2a, + RNase), providing additional

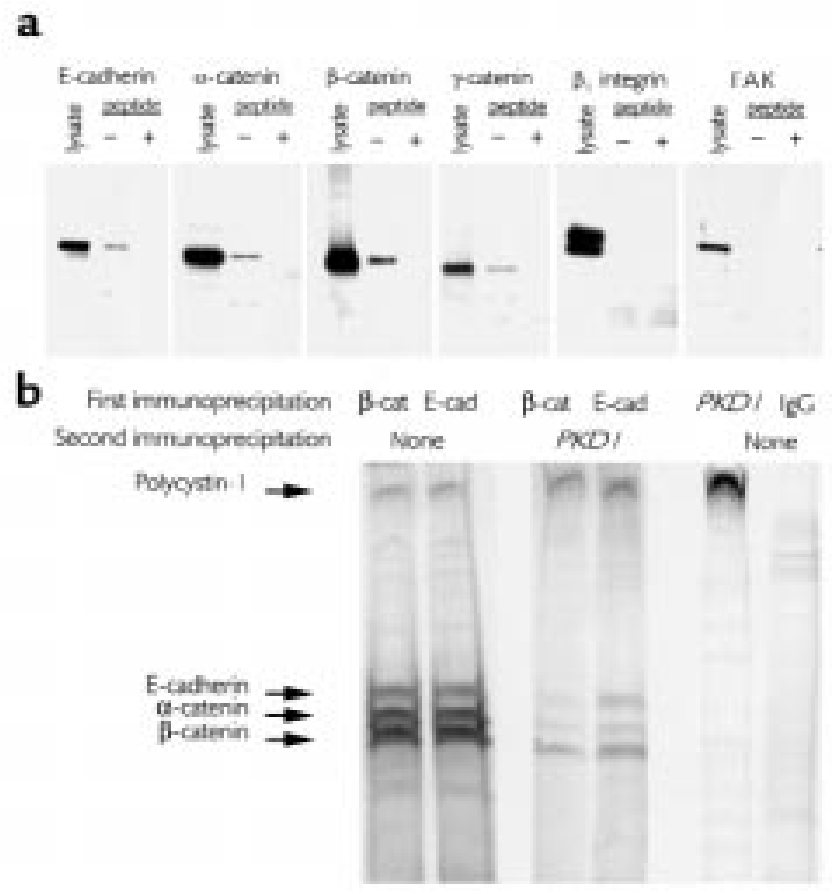

Figure 3

Coimmunoprecipitation of polycystin-1 with junctional complex proteins. (a) Immunoprecipitation of HPAC cell lysates with affinity-purified antibody to polycystin-1. Each panel is an immunoblot probed with antibody to the protein named at the top of the panel. In each panel, the left lane is an aliquot of the cell lysate (lysate). The middle and right lanes were immunoprecipitated with affinity-purified antibody against polycystin-1 in the absence (-) or presence $(+)$ of the immunizing peptide. Each set of samples was probed with antibody to E-cadherin, $\alpha$-catenin, $\beta$-catenin, $\gamma$-catenin, $\beta_{1}$-integrin, or FAK. Note that E-cadherin and the catenins coprecipitated with polycystin- 1 only in the absence of competing peptide. The doublet of $120 \mathrm{kDa}$ and $140 \mathrm{kDa}$ in the lysate probed with antibody to $\beta_{1}$-integrin represents reduced (140 kDa) and unreduced $(120 \mathrm{kDa}) \beta_{1}$-integrin. Results are representative of at least 5 independent experiments. (b) Double immunoprecipitation of metabolically labeled HPAC lysates with antibodies to E-cadherin or $\beta$-catenin (first immunoprecipitation) followed by affinity-purified antibody to polycystin-1 (second immunoprecipitation). The left pair of lanes shows the first immunoprecipitation with monoclonal antibody to $\beta$-catenin ( $\beta$-cat) or E-cadherin (E-cad). Note the high-molecular-weight polypeptide that is precipitated as part of the complex. The center pair of lanes shows the reimmunoprecipitation of the complex with affinity-purified antibody to polycystin-1. The high-molecular-weight polypeptide seen in the left panels was reprecipitated by polycystin antibody and is therefore polycystin-1. Note that some of the E-cadherin/catenin complex remains associated with polycystin-1 (arrows). The right pair of lanes shows control experiments performed on aliquots of the same HPAC lysate. The lane marked "PKD1" was precipitated with affinity-purified antibody to polycystin-1. The lane marked "IgG" was precipitated with the same concentration of nonimmune rabbit anti-mouse IgG. This figure is a single gel. The results are representative of 3 independent experiments. 


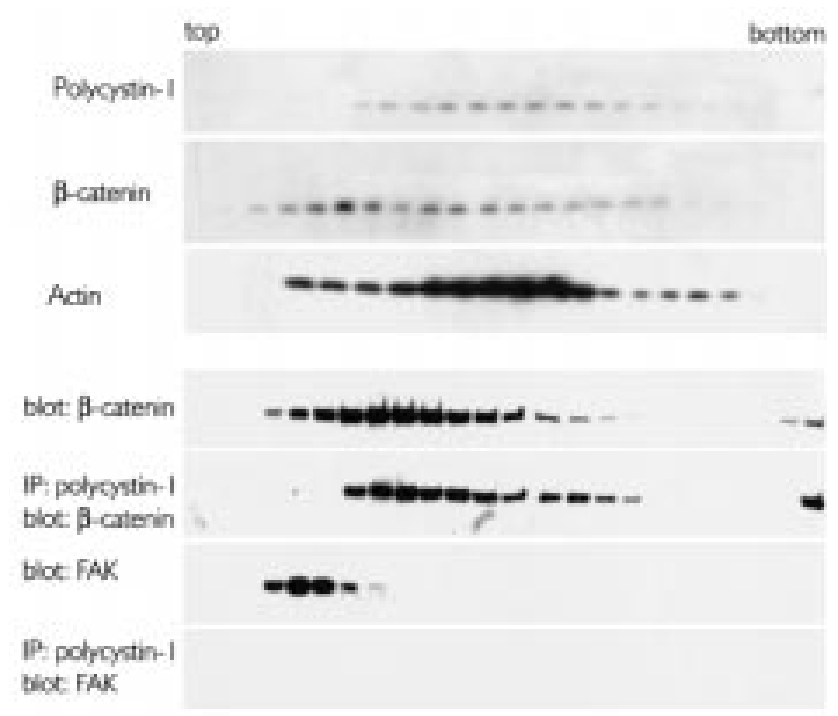

Figure 4

Sucrose density gradient centrifugation of HPAC cell lysates. Top: Distribution of polycystin-1, $\beta$-catenin, and actin within the gradient. Polycystin- 1 was detected by immunoprecipitation and Phosphorlmager analysis (Molecular Dynamics). Immunoblotting was used to detect $\beta$-catenin and actin. Results are representative of 3 independent experiments. Bottom: Distribution of $\beta$-catenin, FAK, and complexes of $\beta$-catenin and polycystin- 1 within gradient. The distributions of $\beta$-catenin and FAK were determined by immunoblotting. The distributions of complexes containing polycystin-1 and either $\beta$-catenin or FAK were determined by immunoprecipitating each fraction of the gradient with affinity-purified antibodies to polycystin- 1 and probing immunoblots of the precipitates with antibodies to $\beta$-catenin (IP: polycystin-1, blot: $\beta$-catenin) or FAK (IP: polycystin-1, blot: FAK). No FAK was coprecipitated with polycystin-1. Results are representative of 3 independent experiments.

evidence that the PCR product was amplified from reverse transcribed $P K D 1$ message.

To discover whether there was significant polycystin-1 protein expression in HPAC cells, confluent cultures were labeled with $\left[{ }^{35} \mathrm{~S}\right]$ methionine/cysteine and lysates were immunoprecipitated with the anti-peptide antibody against polycystin- 1 . Figure $2 \mathrm{~b}$ shows that the immune serum precipitated a polypeptide of approximately $500 \mathrm{kDa}$ that was not precipitated by the preimmune serum. Incubating the immune serum with lysate in the presence of the immunizing peptide blocked precipitation of this polypeptide, showing that the immunoprecipitation was specific for the peptide. Identical results were obtained with 2 different antibodies raised against the extracellular domain of polycystin-1 (not shown). These results show that the HPAC cells express polycystin-1, and that the anti-peptide antibody specifically precipitates polycystin-1 from HPAC cell lysates. We were unable to detect polycystin-1 by immunoblotting lysates of the HPAC cell line, suggesting that polycystin- 1 is expressed at low levels in these cells or that the efficiency of electroblotting this large membrane protein is very low, or both.

E-cadherin and the catenin are coprecipitated with polycystin1. We precipitated HPAC cell lysates with antibody to polycystin- 1 and probed the precipitates with antibodies to Ecadherin and $\alpha-, \beta$-, and $\gamma$-catenin. We found that
E-cadherin and the catenins were coprecipitated with polycystin-1 (Figure 3a, - peptide). Adding B145 peptide to the immunoprecipitation reaction blocked the precipitation of E-cadherin and the catenins (Figure 3a, + peptide), demonstrating that the coprecipitation of E-cadherin and the catenins was specific for the precipitation of polycystin-1. These data show that polycystin-1 interacts with a complex containing E-cadherin and the catenins.

To determine whether polycystin-1 was coprecipitated with E-cadherin and $\beta$-catenin, we performed double immunoprecipitation (Figure 3b). Antibodies to either E-cadherin or $\beta$-catenin (2 left lanes) precipitated a complex containing a high-molecular-weight polypeptide as well as E-cadherin, $\alpha$-catenin, and $\beta$-catenin. The molecular weight of this polypeptide was the same as that of precipitated polycystin-1 (fifth lane, PKD1). Reprecipitation of the complex with anti-polycystin- 1 antibodies showed that the high-molecular-weight polypeptide was polycystin-1 (center pair of lanes). Note that a small fraction of the E-cadherin/catenin complex was reprecipitated with antibody to polycystin-1 despite treatment of the precipitated proteins with $1 \%$ SDS. From densitometric analysis, we determined that only $6.0 \pm 0.5 \%(n=$ 3 ) of precipitable E-cadherin or $\beta$-catenin complexes contained polycystin-1.

Polycystin-1 comigrates with $\beta$-catenin in sucrose density gradients. The coprecipitation of polycystin-1 with E-cadherin and the catenins suggested that these proteins were in a macromolecular complex. Both the colocalization data and the coimmunoprecipitation data showed that only a fraction of E-cadherin/catenin complexes contained polycystin-1. To characterize this complex further, we subjected HPAC cell lysates to sucrose density centrifugation. We chose to use $\beta$-catenin as a marker for the E-cadherin/catenin complex because antibodies to $\beta$ catenin gave the strongest signal in coprecipitation experiments and because $\beta$-catenin metabolism is a major determinant of cell proliferation. Polycystin-1 migrated in a fairly broad range near the center of the gradient (Figure 4, top). The distribution of $\beta$-catenin was broader than that of polycystin-1, extending into lighter fractions of the gradient. The distribution of polycystin-1 overlapped with that of $\beta$-catenin only in the denser parts of the $\beta$-catenin range. As anticipated, polycystin- 1 coprecipitated $\beta$-catenin only in those fractions where polycystin- 1 was present (Figure 4, bottom). The distribution of the complex containing polycystin- 1 and $\beta$-catenin was not homogeneous. The amount of $\beta$-catenin coprecipitated with polycystin- 1 was much greater in the lighter part of the polycystin-1 distribution. This skewed distribution of polycystin-catenin complexes was seen in 4 of 4 independent experiments. These data provide additional evidence that polycystin- 1 is in a complex containing $\beta$-catenin. These results are consistent with both the immunohistochemical and coprecipitation data, which showed that polycystin- 1 and $\beta$ catenin were not completely colocalized or coprecipitated. The data suggest that there are 2 pools of polycystin-1, only 1 of which interacts with a complex containing $\beta$-catenin.

To discover whether the actin cytoskeleton could participate in the formation of a unique complex containing polycystin- 1 and $\beta$-catenin, we examined the distribution of actin within the gradient (Figure 4). Actin comigrated 
with polycystin-1, although the distribution of actin was broader than that of polycystin-1. The peak distribution of polycystin-1 in the gradient matched the peak distribution of actin. These results suggest that actin could be present in polycystin-1 complexes. The distribution of actin overlapped, but did not precisely mimic the distribution of $\beta$-catenin. This result is not surprising, because $\beta$-catenin is found both in complexes containing E-cadherin and in separate complexes containing APC, glycogen synthase kinase, and axin.

Polycystin-1 colocalizes with $\beta_{1}$ integrins but not with FAK. Although the majority of polycystin-1 was concentrated in the apical and lateral aspects of the tubular epithelial cells, there was consistently a small amount of peribasal staining that did not colocalize with E-cadherin or $\alpha$-, $\beta$-, or $\gamma$-catenin. We had previously found that epithelial cells from the cpk mouse model of autosomal recessive polycystic kidney disease had increased $\beta_{1}$-integrin-mediated adhesion to collagen and laminin (37). We speculated that polycystin-1 might interact with both E-cadherin and integrin-type cell adhesion complexes. Accordingly, we performed double-label immunofluorescence with antibodies against the human $\beta_{1}$-integrin subunit or against FAK, the well characterized signal transduction molecule of the focal adhesion. We found that the $\beta_{1}$-integrin subunit was not polarized in these primitive renal epithelia, and that it colocalized with polycystin-1 (Figure 5a). FAK was detected only in the branching tubules of the ureteric bud, which also expressed polycystin-1. FAK was confined to the basal plasma membrane in these epithelia, as was paxillin, another protein of the focal adhesion (data not shown). However, FAK did not colocalize with polycystin1 (Figure $5 \mathrm{~b}$ ). These data suggest that, although polycystin could be present in a complex with a $\beta_{1}$-integrin, it is not present in focal adhesions containing FAK and paxillin.

Polycystin-1 does not coprecipitate or comigrate with FAK or $\beta_{1-}$ integrin. To discover if a small proportion of FAK interacted with polycystin-1, we probed polycystin-1 immuno- precipitates with antibodies to $\beta_{1}$-integrin and FAK. We found that neither $\beta_{1}$-integrin nor FAK were coprecipitated with polycystin-1 (Figure 3). Paxillin was precipitated nonspecifically by agarose beads despite extensive preclearing, so we could not assess coprecipitation of paxillin with polycystin-1. In sucrose density gradient centrifugation, the distribution of FAK overlapped minimally with that of polycystin-1 (Figure 4, bottom). FAK did not coprecipitate with polycystin- 1 in fractions isolated by sucrose density gradient centrifugation (Figure 4, bottom). These data show that complexes containing FAK do not contain polycystin-1, and that polycystin- 1 is not part of the focal adhesion. The coprecipitation data suggest that, if there is an interaction between polycystin- 1 and $\beta_{1}$ integrins, the interaction is not strong. The observation that neither $\beta_{1^{-}}$ integrin nor FAK could be coprecipitated with polycystin1 antibodies provides additional evidence that the coprecipitation of E-cadherin and the catenins by polycystin-1 antibodies was specific.

\section{Discussion}

We have demonstrated that polycystin- 1 is present in complexes containing E-cadherin and the catenins. E-cadherin and the catenins regulate cell proliferation, cell polarity, and tissue morphogenesis. In ADPKD, the cysts develop from tubules, a striking example of abnormal morphogenesis. The creation of this abnormal structure, the cyst, is accompanied by perturbations in cell proliferation and cell polarity. Our discovery that polycystin- 1 interacts with E-cadherin and $\alpha$-, $\beta$-, and $\gamma$-catenin raises obvious questions about whether polycystin- 1 affects the assembly of junctional complexes or signaling from these complexes. Because the catenins have effects on such diverse cellular functions as the polarization of the cytoskeleton (18), regulation of gene transcription (38), and the formation of desmosomes $(39,40)$, the range of possibilities is large, but is consistent with the protean manifestations of ADPKD.

One of the first questions that arises from our work is
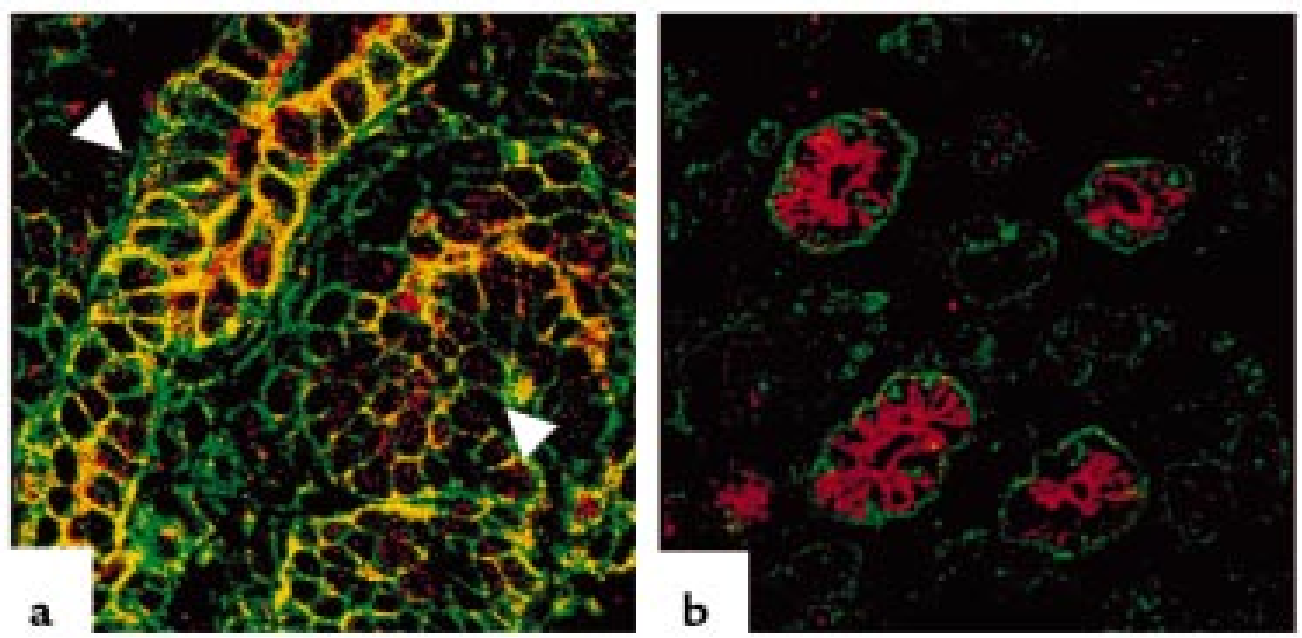

\section{Figure 5}

Confocal images of sections of human fetal kidney stained with antibody to polycystin-1 (red) and either $\beta_{1}$-integrin (a) or FAK (green) (b). Only the dual-scanned images are shown. The areas where polycystin- 1 colocalized with $\beta_{1}$-integrin (a) are yellow. Polycystin- 1 was expressed only in the epithelial structures of the ureteric bud and the S-shaped body (arrowheads), whereas $\beta_{1}$-integrin was ubiquitously expressed in both the epithelia (yellow staining due to colocalization with polycystin-1) and the surrounding mesenchyme (green staining, because polycystin-1 is not expressed in the mesenchyme). The absence of yellow staining in (b) shows that polycystin-1 did not colocalize with FAK. 
whether polycystin-1 interacts directly with E-cadherin or with 1 of the catenins. Our data do not allow us to distinguish between a direct or an indirect interaction. Yeast 2-hybrid analysis conducted in several labs did not identify E-cadherin or any of the catenins as binding partners for the carboxyl terminal domain of polycystin-1 (41-45), nor have preliminary biochemical experiments identified any of these proteins in pull-down assays from epithelial cell lysates (van Adelsberg, Huan, and Walz, unpublished observations). Polycystin-1 is a polytopic integral membrane protein. Current models of its structure favor 11 transmembrane domains with 5 intracellular loops. Any of these loops could bind directly to Ecadherin, to a catenin, or to an adapter molecule that could provide a bridge between polycystin- 1 and the E-cadherin/catenin complex. $\beta$-catenin and $\gamma$-catenin bind directly to $\alpha$-catenin, giving rise to 2 distinct cadherin-catenin complexes $(46,47)$. Polycystin- 1 interacts with both of these complexes. The most parsimonious explanation is that polycystin-1 (or the hypothetical adapter) interacts with E-cadherin or with $\alpha$-catenin, which are common to both complexes.

We found that the interaction between polycystin-1 and the E-cadherin/catenin complex was not stoichiometric. The colocalization data showed separate areas of polycystin-1 and cadherin/catenin staining, suggesting that polycystin-1 interacts only with a discrete pool of cadherin and catenins. Sucrose density gradient centrifugation also identified a pool of polycystin- 1 that did not interact with $\beta$-catenin. These data suggest that the interaction of polycystin-1 with the E-cadherin/catenin complex could be regulated. Complexes containing E-cadherin, the catenins, and polycystin-1 might have a specialized role in signal transduction.

What is the role of an interaction between polycystin-1 and the E-cadherin/catenin complex? One possibility is that polycystin-1 is involved in the formation of the E-cadherin/catenin complex. This hypothesis appears unlikely, because PKD1 null mice die during late organogenesis, not at the peri-implantation stage as do E-cadherin or $\beta$-catenin null mice (48-51). Preliminary work (Huan and van Adelsberg, unpublished observations) suggests that E-cadherin/catenin complexes are intact in cyst-lining epithelial cells isolated from ADPKD kidneys.

The interaction of polycystin-1 with the E-cadherin/catenin complex could be involved in correct targeting of polycystin-1. Mutations that affect targeting rather than biochemical activity are well documented. One well characterized example is the $\Delta \mathrm{F} 508$ mutation of the cystic fibrosis transmembrane conductance regulator (CFTR) ion channel, which produces a functional channel that is not targeted to the plasma membrane (52). Retention of targeted proteins in the correct plasma membrane domain is one mechanism for maintaining cell polarity. This mechanism has been well characterized for $\mathrm{Na}, \mathrm{K}$-ATPase. This protein interacts directly with ankyrin $(27,53)$, which is complexed with nonerythrocyte spectrin (fodrin), and then, through actin and $\alpha$-catenin, to the E-cadherin/catenin complex $(24,33,34,54,55)$. The hypothesis that the interaction between polycystin-1 and the E-cadherin/catenin complex stabilizes correctly polarized polycystin-1 in a manner analogous to that of $\mathrm{Na}, \mathrm{K}$ ATPase predicts several results. First, the half-life of mutant polycystin-1 in ADPKD cyst-lining epithelial cells should be reduced. Second, the complex should contain cytoskeletal elements like actin and possibly ankyrin or spectrin. We found that polycystin-1 and actin comigrated in sucrose gradients, suggesting that they could be part of a complex. Two of the candidate proteins that interact with the carboxyl terminal domain of polycystin- 1 contain spectrin repeats, suggesting that polycystin-1 could interact with spectrin or fodrin. Third, polycystin-1 should be incorrectly targeted in cyst-lining epithelial cells of ADPKD. A corollary to the hypothesis is that only some mutations in PKD1 would disrupt the interaction of polycystin-1 with E-cadherin and the catenins, whereas other mutations might cause different effects on polycystin-1 processing or function. Apparent mislocalization of polycystin-1 has been observed in at least 1 case of ADPKD. These data support the idea that the interaction of E-cadherin/catenin complex with polycystin-1 could be involved in polycystin-1 targeting.

A third possible role for the interaction of polycystin-1 with E-cadherin/catenin complexes is that of modulating signaling from these complexes, in particular by effects on $\beta$-catenin metabolism. This is an attractive hypothesis for a variety of reasons. First, expression of the carboxyl terminal tail of polycystin- 1 as a membranetargeted chimera protected cytoplasmic $\beta$-catenin from degradation by the proteasome and increased transcription from a reporter for Siamois, a genuine $\beta$-catenin target (56). Second, high expression of $c-m y c$, a likely $\beta$-catenin target, is found in both human ADPKD cystlining epithelial cells and cyst-lining epithelial cells from 2 rodent models of polycystic kidney disease (57-59). Transgenic mice that overexpress $c$-myc in renal epithelial cells develop fatal polycystic kidney disease, demonstrating that $c-m y c$ is in the pathway to cyst formation (60). Third, nuclear $\beta$-catenin staining is found in the $b c l$ 2 null mouse model of polycystic kidney disease (61). The interaction of $\beta$-catenin with LEF transcription factors is known to target $\beta$-catenin to the nucleus (62). These data suggest that aberrant $\beta$-catenin signaling could be a common feature of polycystic kidney diseases. This hypothesis predicts that the half-life of $\beta$-catenin will be prolonged in cyst-lining epithelial cells in polycystic kidney disease, and that expression of other $\beta$-catenin target genes will be upregulated in these cells.

Our data show that polycystin-1 does not interact directly with focal adhesions in the steady state that is characteristic of epithelial cells in tissue or confluent cultures. We had previously observed increased $\beta_{1}$-integrin-mediated adhesion to extracellular matrix in cells isolated from a mouse model of polycystic kidney disease, suggesting that defects in cell-matrix interaction might contribute to cyst formation (37). Our new data suggest that this phenotype is unlikely to be caused by a direct effect of polycystin-1 on focal adhesions, but could be an example of cross-talk between cadherins and integrin $(63,64)$. We had found that the integrin that mediates increased matrix adhesion in cpk mouse epithelial cells is $\alpha_{1} \beta_{1}$-integrin (van Adelsberg and Almonte, 
unpublished observations). Expression of this integrin is upregulated in autosomal recessive polycystic kidney disease and in ADPKD (65), suggesting that upregulation of $\alpha_{1} \beta_{1}$-integrin-mediated adhesion is part of a common pathway in cyst formation. This hypothesis would place the regulation of $\alpha_{1} \beta_{1}$-integrin expression downstream of the PKD and ARPKD genes.

Our observation that $\beta_{1}$ integrins are not polarized during renal development is not new (66-68). However, we are the first to describe the localization of FAK and paxillin in developing kidney. Our data show that paxillin and FAK are confined to the basal plasma membrane. The $\beta_{1}$ integrins, however, are basal, lateral, and apical. This result suggests that either these apical and lateral integrins are not involved in signaling or that other signaling complexes containing integrins but not the classic molecules of the focal adhesion are present in renal epithelial cells. Because Madin-Darby canine kidney (MDCK) cells express apical $\beta_{1}$ integrins and respond to them by remodeling the axis of apicobasal polarity when collagen is overlaid on an MDCK monolayer $(69,70)$, it seems likely that the apical $\beta_{1}$ integrins are functional.

\section{Acknowledgments}

Janet van Adelsberg is an Established Investigator of the American Heart Association. This project was funded in part by grant 1-FY97-0513 from the March of Dimes. Confocal microscopy was performed at the Confocal Microscopy Facility of Columbia University, which was established by National Institutes of Health (NIH) Shared Instrumentation Grant 1S10-RR10406 and is supported by NIH grant 5-P30-CA13696 as part of the Herbert Irving Cancer Center at Columbia University. We are grateful to Theresa Swayne of the Confocal Microscopy Facility for her invaluable assistance in obtaining the confocal images shown here, and to Vivette D'Agati and Llewellyn Ward of the Department of Pathology at Columbia University for their help in obtaining and analyzing the kidney sections shown here.

1. Gabow, P.A. 1993. Autosomal dominant polycystic kidney disease. $N$. Engl. J. Med. 329:332-342.

2. European Polycystic Kidney Disease Consortium. 1994. The polycystic kidney disease 1 gene encodes a $14 \mathrm{~kb}$ transcript and lies within a duplicated region on chromosome 16. Cell. 77:881-894.

3. American PKD1 Consortium. 1995. Analysis of the genomic sequence for the autosomal dominant polycystic kidney disease gene predicts the presence of a leucine-rich repeat. Hum. Mol. Genet. 4:575-582.

4. International Polycystic Kidney Disease Consortium. 1995. Polycystic kidney disease: the complete structure of the PKD1 gene and its protein. Cell. 81:289-298.

5. Hughes, J., et al. 1995. The polycystic kidney disease 1 gene encodes a novel protein with multiple cell recognition domains. Nat. Genet. 10:151-159.

6. Ariza, M., et al. 1997. A family with a milder form of adult dominant polycystic kidney disease not linked to the PKD1 (16p) or PKD2 (4q) genes. J. Med. Genet. 34:587-589.

7. Daoust, M.C., Reynolds, D.M., Bichet, D.G., and Somlo, S. 1995. Evidence for a third genetic locus for autosomal dominant polycystic kidney disease. Genomics. 25:733-736.

8. de Almeida, S., et al. 1995. Autosomal dominant polycystic kidney disease: evidence for the existence of a third locus in a Portuguese family. Hum. Genet. 96:83-88.

9. Turco, A.E., Clementi, M., Rossetti, S., Tenconi, R., and Pignatti, P.F. 1996. An Italian family with autosomal dominant polycystic kidney disease unlinked to either the PKD1 or PKD2 gene. Am. J. Kidney Dis. 28:759-761.

10. Qian, F., et al. 1997. PKD1 interacts with PKD2 through a probable coiled-coil domain. Nat. Genet. 16:179-183.
11. Tsiokas, L., Kim, E., Arnould, T., Sukhatme, V.P., and Walz, G. 1997. Homo- and heterodimeric interactions between the gene products of PKD1 and PKD2. Proc. Natl. Acad. Sci. USA. 94:6965-6970.

12. van Adelsberg, J.S., Chamberlain, S., and D'Agati, V. 1997. Polycystin expression is temporally and spatially regulated during renal development. Am. J. Physiol. 272:F602-F609.

13. Palsson, R., et al. 1996. Immunolocalization of PKD1 in normal kidneys. Mol. Med. 2:702-711.

14. Ibraghimov-Beskrovnaya, O., et al. 1997. Polycystin: in vitro synthesis, in vivo tissue expression, and subcellular localization identifies a large membrane-associated protein. Proc. Natl. Acad. Sci. USA. 94:6397-6402.

15. Ong, A.C., Ward, C.J., Biddolph, S., Migone, N., and Harris, P.C. 1997. Polycystin expression in PKD1, infantile PKD1, and TSC-2/PKD1 cystic kidney: evidence against a two-hit disease mechanism in cyst initiation. J. Am. Soc. Nephrol. 8:378A. (Abstr.)

16. Griffin, M.D., et al. 1997. Expression of polycystin in mouse metanephros and extra-metanephric tissues. Kidney Int. 52:1196-1205.

17. Geng, L., et al. 1996. Identification and localization of polycystin, the PKD1 gene product. J. Clin. Invest. 98:2674-2682.

18. Nelson, W.J. 1993. Regulation of cell surface polarity in renal epithelia. Pediatr. Nephrol. 7:599-604.

19. Gumbiner, B.M., and McCrea, P.D. 1993. Catenins as mediators of the cytoplasmic functions of cadherins. J. Cell Sci. Suppl. 17:155-158.

20. Aberle, H., Schwartz, H., and Kemler, R. 1996. Cadherin-catenin complex: protein interactions and their implications for cadherin function. J. Cell Biochem. 61:514-523.

21. Gumbiner, B. 1995. Signal transduction by $\beta$-catenin. Curr. Opin. Cell Biol. 7:634-640.

22. Barth, A.I., Nathke, I.S., and Nelson, W.J. 1997. Cadherins, catenins and APC protein: interplay between cytoskeletal complexes and signaling pathways. Curr. Opin. Cell Biol. 9:683-690.

23. Grantham, J.J. 1990. Polycystic kidney disease: neoplasia in disguise. Am. J. Kidney Dis. 15:110-116.

24. Nelson, W.J., Shore, E.M., Wang, A.Z., and Hammerton, R.W. 1990. Identification of a membrane-cytoskeletal complex containing the cell adhesion molecule uvomorulin (E-cadherin), ankyrin, and fodrin in MadinDarby canine kidney epithelial cells. J. Cell Biol. 110:349-357.

25. McNeill, H., Ozawa, M., Kemler, R., and Nelson, W.J. 1990. Novel function of the cell adhesion molecule uvomorulin as an inducer of cell surface polarity. Cell. 62:309-316.

26. Graeve, L., Drickamer, K., and Rodriguez-Boulan, E. 1989. Polarized endocytosis by Madin-Darby canine kidney cells transfected with functional chicken liver glycoprotein receptor. J. Cell Biol. 109:2809-2816.

27. Devarajan, P., Scaramuzzino, D.A., and Morrow, J.S. 1994. Ankyrin binds to two distinct cytoplasmic domains of Na,K-ATPase alpha subunit. Proc. Natl. Acad. Sci. USA. 91:2965-2969.

28. Alejandro, V.S., et al. 1995. Postischemic injury, delayed function and $\mathrm{Na}^{+} / \mathrm{K}(+)$-ATPase distribution in the transplanted kidney. Kidney Int. 48:1308-1315.

29. Molitoris, B.A., Geerdes, A.M., and McIntosh, J.R. 1991. Dissociation and redistribution of $\mathrm{Na}^{+}, \mathrm{K}(+)$-ATPase from its surface membrane actin cytoskeletal complex during cellular ATP depletion. J. Clin. Invest. 88:462-469.

30. Wilson, P.D., et al. 1991. Reversed polarity of $\mathrm{Na}(+)-\mathrm{K}(+)$-ATPase: mislocation to apical plasma membranes in polycystic kidney disease epithelia. Am. J. Physiol. 260:F420-F430.

31. Brill, S.R., et al. 1996. Immunolocalization of ion transport proteins in human autosomal dominant polycystic kidney disease. Proc. Natl. Acad. Sci. USA. 93:10206-10211.

32. Carone, F.A., et al. 1994. Cell polarity in human renal cystic disease. Lab. Invest. 70:648-655.

33. Ozawa, M., and Kemler, R. 1992. Molecular organization of the uvomorulin-catenin complex. J. Cell Biol. 115:989-996.

34. Jou, T.S., Stewart, D.B., Stappert, J., Nelson, W.J., and Marrs, J.A. 1995. Genetic and biochemical dissection of protein linkages in the cadherincatenin complex. Proc. Natl. Acad. Sci. USA. 92:5067-5071.

35. van Adelsberg, J.S. 1999. The role of the polycystins in kidney development. Pediatr. Nephrol. 13:454-459.

36. Lu, W., et al. 1997. Perinatal lethality with kidney and pancreas defects in mice with a targeted Pkd1 mutation. Nat. Genet. 17:179-181.

37. van Adelsberg, J.S. 1994. Murine polycystic kidney epithelial cell lines have increased integrin-mediated adhesion to collagen. Am. J. Physiol. 276:F1082-F1093.

38. Bullions, L.C., and Levine, A.J. 1998. The role of beta-catenin in cell adhesion, signal transduction, and cancer. Curr. Opin. Oncol. 10:81-87.

39. Marrs, J.A., et al. 1995. Plasticity in epithelial cell phenotype: modulation by expression of different cadherin cell adhesion molecules. J. Cell Biol. 129:507-519.

40. Lewis, J.E., et al. 1997. Cross-talk between adherens junctions and desmosomes depends on plakoglobin. J. Cell Biol. 136:919-934.

41. Qian, F., Germino, J., Zhang, F., and Germino, G. 1997. Putative binding partners of polycystin. J. Am. Soc. Nephrol. 8:380A. (Abstr.) 
42. Maeda, Y., et al. 1997. Identification of potential binding proteins for PKD2.J. Am. Soc. Nephrol. 8:376A. (Abstr.)

43. Kim, E., Arnould, T., and Walz, G. 1997. Isolation of polycystin-interacting proteins. J. Am. Soc. Nephrol. 8:375A. (Abstr.)

44. Kim, E., et al. 1999. Interaction between RGS7 and polycystin. Proc. Natl. Acad. Sci. USA. 96:6371-6376.

45. Kim, K., Sharma, C.P., and Arnaout, M.A. 1997. An interaction of 14-33 adapter proteins with polycystin. J. Am. Soc. Nephrol. 8:375A. (Abstr.)

46. Hinck, L., Näthke, I.S., Papkoff, J., and Nelson, W.J. 1994. Dynamics of cadherin/catenin complex formation: novel protein interactions and pathways of complex assembly. J. Cell Biol. 125:1327-1340.

47. Näthke, I.S., Hinck, L., Swedlow, J.R., Papkoff, J., and Nelson, W.J. 1994. Defining interactions and distributions of cadherin and catenin complexes in polarized epithelial cells. J. Cell Biol. 125:1341-1352.

48. Larue, L., Ohsugi, M., Hirchenhain, J., and Kemler, R. 1994. E-cadherin null mutant embryos fail to form a trophectoderm epithelium. Proc. Natl. Acad. Sci. USA. 91:8263-8267.

49. Riethmacher, D., Brinkmann, V., and Birchmeier, C. 1995. A targeted mutation in the mouse E-cadherin gene results in defective preimplantation development. Proc. Natl. Acad. Sci. USA. 92:855-859.

50. Levine, E., Lee, C.H., Kintner, C., and Gumbiner, B.M. 1994. Selective disruption of E-cadherin function in early Xenopus embryos by a dominant negative mutant. Development. 120:901-909.

51. Haegel, H., et al. 1995. Lack of beta-catenin affects mouse development at gastrulation. Development. 121:3529-3537.

52. Kopito, R.R. 1999. Biosynthesis and degradation of CFTR. Physiol. Rev. 79:S167-S173.

53. Jordan, C., Puschel, B., Koob, R., and Drenckhahn, D. 1995. Identification of a binding motif for ankyrin on the alpha-subunit of $\mathrm{Na}^{+}, \mathrm{K}(+)-$ ATPase. J. Biol. Chem. 270:29971-29975.

54. McCrea, P.D., Turck, C.W., and Gumbiner, B.M. 1991. A homolog of the armadillo protein in Drosophila (plakoglobin) associated with E-cadherin. Science. 254:1359-1361.

55. Hirano, S., Kimoto, N., Shimoyama, Y., Hirohashi, S., and Takechi, M. 1992. Identification of a neural $\alpha$-catenin as a key regulator of cadherin function and multicellular organization. Cell. 70:293-301.

56. Kim, E., et al. 1999. The polycystic kidney disease 1 gene product modulates Wnt signaling. J. Biol. Chem. 274:4947-4953.

57. Cowley, B.D., Jr., Smardo, F.L., Jr., Grantham, J.J., and Calvet, J.P. 1987.
Elevated c-myc protooncogene expression in autosomal recessive polycystic kidney disease. Proc. Natl. Acad. Sci. USA. 84:8394-8398.

58. Klingel, et al. 1992. Expression of differentiation antigens and growthrelated genes in normal kidney, autosomal dominant polycystic kidney disease, and renal cell carcinoma. Am. J. Kidney Dis. 19:22-30.

59. Cowley, B.D., Jr., et al. 1993. Autosomal-dominant polycystic kidney disease in the rat. Kidney Int. 43:522-534.

60. Trudel, M., D'Agati, V.D., and Costantini, F. 1991. C-myc as an inducer of polycystic kidney disease in transgenic mice. Kidney Int. 39:665-671.

61. Sorenson, C.M. 1999. Nuclear localization of beta-catenin and loss of apical brush border actin in cystic tubules of bcl-2-/- mice. Am. J. Physiol. 276:F210-F217.

62. Huber, O., et al. 1996. Nuclear localization of beta-catenin by interaction with transcription factor LEF-1. Mech. Dev. 59:3-10.

63. Monier-Gavelle, F., and Duband, J.L. 1997. Cross talk between adhesion molecules: control of $\mathrm{N}$-cadherin activity by intracellular signals elicited by beta 1 and beta3 integrins in migrating neural crest cells. J. Cell Biol. 137:1663-1681.

64. Novak, A., et al. 1998. Cell adhesion and the integrin-linked kinase regulate the LEF-1 and beta-catenin signaling pathways. Proc. Natl. Acad. Sci. USA. 95:4374-4379.

65. Daikha-Dahmane, F., et al. 1997. Distribution of alpha-integrin subunits in fetal polycystic kidney diseases. Pediatr. Nephrol. 11:267-273.

66. Korhonen, M., Ylanne, J., Laitinen, L., and Virtanen, I. 1990. The alpha 1-alpha 6 subunits of integrins are characteristically expressed in distinct segments of developing and adult human nephron. J. Cell Biol. 111:1245-1254.

67. Simon, E.E., and McDonald, J.A. 1990. Extracellular matrix receptors in the kidney cortex. Am. J. Physiol. 259:F783-F792.

68. Korhonen, M., Laitinen, L.,Ylänne, J., Gould, V.E., and Virtanen, I. 1992. Integrins in developing, normal, and malignant human kidney. Kidney Int. 41:641-644.

69. Schwimmer, R., and Ojakian, G.K. 1995. The alpha 2 beta 1 integrin regulates collagen-mediated MDCK epithelial membrane remodeling and tubule formation. J. Cell Sci. 108:2487-2498.

70. Zuk, A., and Matlin, K.S. 1996. Apical beta 1 integrin in polarized MDCK cells mediates tubulocyst formation in response to type I collagen overlay. J. Cell Sci. 109:1875-1889. 